\title{
Téoros
}

Revue de recherche en tourisme

\section{Le point de vue des intervenants}

\section{Lynda Johnson et Jacques Luneau}

Volume 1, numéro 1, février 1982

L’hospitalité au Québec

URI : https://id.erudit.org/iderudit/1080862ar

DOI : https://doi.org/10.7202/1080862ar

Aller au sommaire du numéro

Éditeur(s)

Université du Québec à Montréal

ISSN

0712-8657 (imprimé)

1923-2705 (numérique)

Découvrir la revue

Citer ce document

Johnson, L. \& Luneau, J. (1982). Le point de vue des intervenants. Téoros, 1(1), 13-14. https://doi.org/10.7202/1080862ar d'utilisation que vous pouvez consulter en ligne.

https://apropos.erudit.org/fr/usagers/politique-dutilisation/ 


\section{Le point de vue des intervenants}

Par Lynda Johnson et Jacques Luneau

Consciente que I'hospitalité est vécue et perçue de diverses façons, l'équipe chargée de l'organisation du Congrès de l'A.T.T. 1981 a songé à recueillir les impressions des différents agents touristiques du Québec.

Les entrevues furent faites à l'échelle du Québec, au printemps 81, tant auprès des intervenants publics et parapublics, organisant l'accueil et publicisant une image régionale, qu'auprès des intervenants privés vivant quotidiennement la relation visiteur-visité.

Ces entrevues individuelles ont fourni des éléments de réponse sur l'impor- tance réelle qu'accordent les intervenants à I'hospitalité, sur la nature des réalisations concrètes et sur les efforts de collaboration et de concertation entre les intervenants et la population.

A la question, "la population québécoise vous semble-t-elle hospitaliere?". la majorité des intervenants s'accordent pour dire qu'elle l'est de plus en plus. Cependant cette relation d'hospitalité s'observe à travers deux vécus fort différents.

En premier lieu, dans les régions où le flux touristique est considérable, il y aura toujours une partie de la popula- tions qui sera refractaire au tourisme. En effet, une affluence de touristes génère une hausse des prix et limite ainsi l'accès à certains sites pour la population locale.

"Il y a donc des conflits entre les gens qui vivent du tourisme et ceux qui n'en veulent tout simplement pas".

(Association touristique régionale de Lanaudière)

En second lieu, dans les régions où le tourisme n'est encore qu'au stade embryonnaire, on observe une faible conscientisation de la population locale face

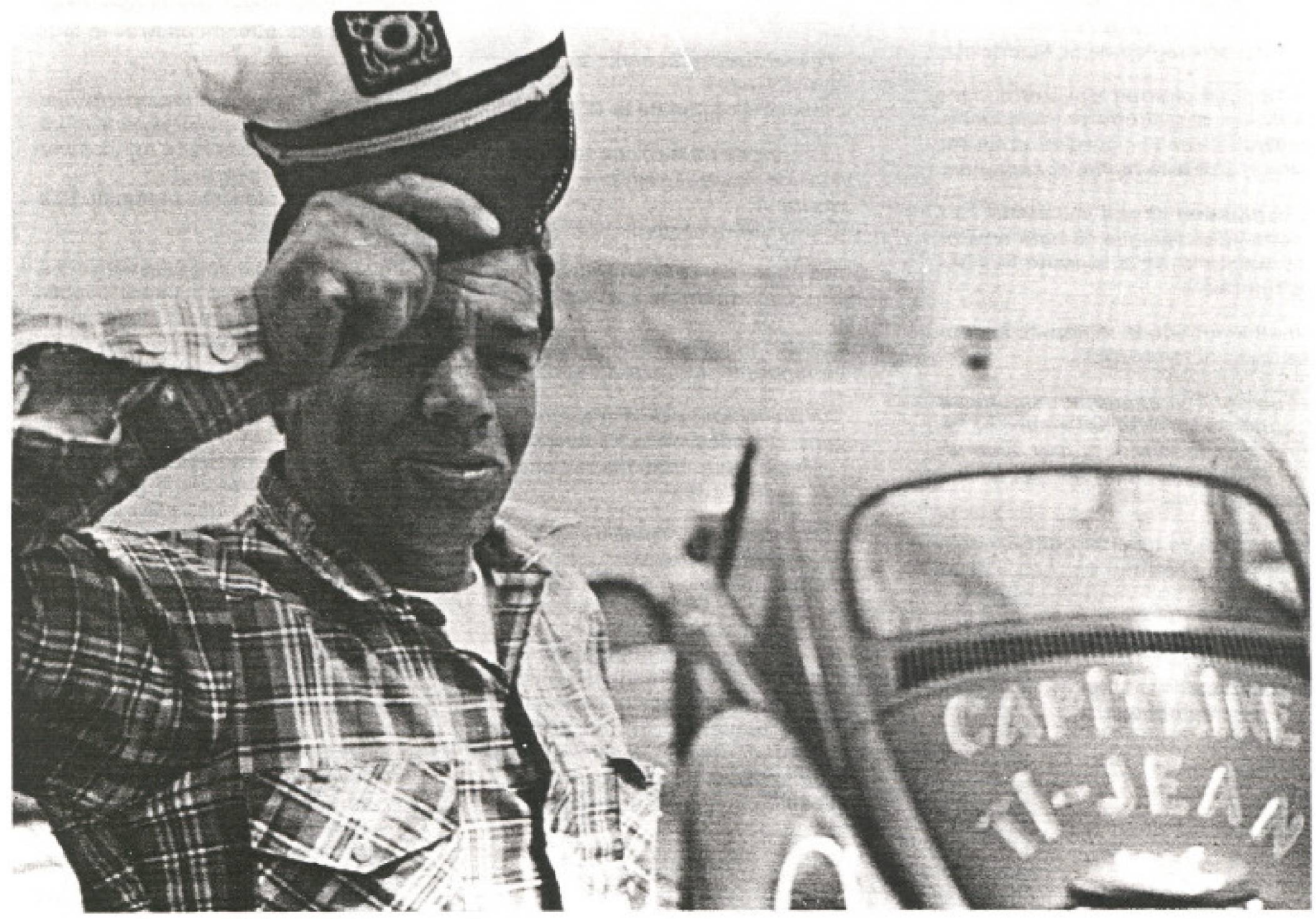


aux bienfaits de cette nouvelle industrie et à la nécessité d'améliorer l'accueil.

L'hospitalité se définit de plusieurs façons. Au niveau de l'idée que les intervenants s'en font, deux perceptions générales se dégagent. L"hospitalité c'est:

1. La continuité d'une tradition, selon laquelle on affirme que la population est toujours aussi hospitaliere.

"L'attitude de la population est positive. il y a une tradition vieille de 100 ans et plus."

(Aubergiste - Cap à l'Aigle, région de Charlevoix)

En ce sens, la pratique continuelle du tourisme dans une région engendre un effort des intervenants pour s'assurer d'un bon accueil de la population locale. Par exemple, certaines régions, comme le Saguenay-Lac St-Jean, la Beauce et I'Estrie ont băti leur image touristique à même cette réputation d'hospitalité.

Par contre, certains intervenants notent que:

"Ia tradition d'hospitalité est moins prononcée, le phénomene familial ayant change, les relations de famille etant moins accentuees, $/ i$ dee que Ion se fait de /hospi= talité, n'est plus la méme."

(Hôtelier = région de la Mauricie)

Ainsi donc, il pourrait s'averer dangereux d'axer des efforts promotionnels uniquement sur l'hospitalité promise. car elle n'a plus la meme connotation.

2. L'expression d'une nécessité, car I'hospitalité est perçue comme un élément majeur dans la réussite des années futures.

Un intervenant de la région de Lanau: dière disait notamment:

"un entrepreneur en tourisme qui ne serait pas chaleureux, ne ferait pas long feu, tout comme un chirurgien qui aurait peur du sang."

et un autre de renchérir que I'hospitalité est très importante.

"les touristes sont avant tout des clients comme les autres qui me font vivre"

(Garagiste - région de I'AbitibiTémiscamingue)
Le fait d'être ou non hospitalier ne s'évalue pas nécessairement en termes monétaires, toutefois l'on est conscient de son impact pour la bonne marche de l'industrie de services.

"Laccueil...c est la place premiere. sinon c'est la faillite."

(Pourvoyeur, région de la CôteNord)

"L'hospitalité ne se vend pas, mais elle doit se refleter globalement." (Bureau des Congres, Ville de Sher = brooke)

$\mathrm{Au}$ niveau pratique, les intervenants ont diverses manières d'être hospitalier: Avant tout, il s'agit

- d'être sociable

II faut tout d'abord faire sentir au touriste qu'il est le bienvenu:

"Bienvenue chez nous, ca me fait plaisir que vous veniez."

(Accompagnateur, région de la Beauce)

"Bonjour! Avez-vous fait bon voyage?"

(Garagiste, Ville de La Sarre, région de ('Abitibi)

Cette sociabilité s'exprime par une foule de gestes et d'attitudes:

"Les accueillir comme si on les connaissait."

(Hôtelier - région de la Côte Nord)

"J'essaie de faire ce quej"aime que ron me lasse, lorsque je suis en voyage."

(Garagiste - région de I'Abitibi)

- d'être en mesre de fournir des informations concernant les attraits de la région.

Informer les touristes est une autre façon de rendre leur sejour agréable:

"La meilleure façon d'accueillir les gens, c'est de bien les renseigner." (Association touristique régionale de Lanaudière)

"On prend le temps de repondre a toutes les questions ${ }^{\text {"* }}$

(Restaurateur - région de la Côte Nord)

- ...et de penser au lendemain

Les intervenants expriment une volonté commune d'amelliorer l'infrastructure d'accueil actuelle. A ce niveau, les programmes de formation des travailleurs en place seraient un moyen d"assurer un niveau de qualitế permanent.
"Il faut entrainer le personnel. C'est de loin la chose la plus importante lorsquion parle d'hospitalite: quand un client est mal accueilli, il ne revient pas."

(Aubergiste - Lanaudière)

"L'accueil est relié au service de l'établissement: il faut que les em. ployes soient qualifies et aient beaucoup d"expérience."

(Restaurateur - région de I'Outa. ouais)

L'information à véhiculer ne doit pas être laissée à chacun, isolement. Une concertation accrue entre les intervenants permettrait de prendre conscience du potentiel touristique quilis ont en commun.

"Il doit y avoir une communication plus intense avec les associations afin d'etudier les besoins des touristes et de connaître ce que lon a d offrir, pour pouvoir diriger le tou. riste vers nous."

(Restaurateur - région de Québec)

L'association de la population au développement du tourisme à l'intérieur des structures régionales et locales intensifierait la qualité de l'accueil. D'une nou= velle perception de I'hospitalité, se dégagerait un dynamisme important dans les relations quotidiennes avec le tourisme.

"On forme des comités, on travaille avec les gens qui prennent alors des responsabilites, c'est comme ca qu'on storganise. ${ }^{* a}$

(Service des loisirs - région du Bas St-Laurent)

De plus, un énorme boulot est encore a faire au niveau de la publicité. II faut développer une image du Québec plus adéquate aux réalités d'ici. A propos de la publicité gouvernementale "warmer up north", un intevenant nous disait précisément que:

"c'est un ingrédient majeur dans toute publicite touristique de n'importe quelle région. Dans ce sens. ce n'est pas tres original."

(Association touristique régionale de Lanaudière)

Unis dans une volonté commune d'améliorer l'accueil au Québec, les intervenants seront alors en mesure d'offrir un produit de qualité pour que:

\footnotetext{
"Au retour d'un voyage au Ouébec. les gens disent: Nous avons rencontré des gens charmants: la nourriture était bonne... cela passe avant le soleil... C'est cela lhospitalite."

(Restaurateur - Ville de Granby, région de I'Estrie)
} 